\title{
COMPUTABLE CONSTRAINTS ON ENTANGLEMENT-SHARING OF MULTIPARTITE QUANTUM STATES
}

\author{
YONG-CHENG OU \\ Physics Department, Southern Illinois University \\ Carbondale, Illinois 62901-4401, USA \\ MARK S. BYRD \\ Physics Department and Computer Science Department, Southern Illinois University \\ Carbondale, Illinois 62901-4401, USA
}

\begin{abstract}
Negativity is regarded as an important measure of entanglement in quantum information theory. In contrast to other measures of entanglement, it is easily computable for bipartite states in arbitrary dimensions. In this paper, based on the negativity and realignment, we provide a set of entanglement-sharing constraints for multipartite states, where the entanglement is not necessarily limited to bipartite and pure states, thus aiding in the quantification of constraints for entanglement-sharing. These may find applications in studying many-body systems.
\end{abstract}

Keywords: Negativity, Realignment, Monogamy, Entanglement

\section{Introduction}

Entanglement is an important resource for quantum information processing (QIP) and is generally believed to be a key resource in quantum algorithms. Although pure entangled states are highly desirable in QIP $[1,2]$, the available states are most often mixed due to noises of various types in real experiments. Consequently, in recent years a great deal of effort has been made to develop methods for detecting, quantifying, and characterizing entanglement of bipartite and multipartite quantum states [3, 4].

Detection and characterization of entanglement are most often based on the concept of separability proposed by Werner [5]. However, while some lower-dimensional bipartite states have been quantified thoroughly, for higher dimensions the Peres-Horodecki criterion $[6,7]$ detects entanglement of many states, but not all. Quantifying entanglement has been even more difficult. For example, one of the most prevalent measures of entanglement, the entanglement of formation (EOF), has an analytical formula only for two-qubit states [8]. Finding the EOF for other systems of states is a challenging and open problem. More recently new methods for describing entangled states have been developed such as the realignment criterion [9, 10], entanglement witnesses [11], covariance matrices approach [12], and improved realignment criterion [13], among others. From these, several useful lower bounds for the concurrence have been derived $[14,15,16,17,18,19,20,21,13,22]$. Some measures of entanglement, though being unable to detect bound entanglement, are relatively easy to calculate.

Furthermore, the quantification of entanglement of multipartite states is even more difficult although the important monogamy nature of entanglement was discovered, which states that the entanglement between the particle $A$ and $B$ constrains that between the particle $A$ and $C$. The monogamy inequalities developed so far, such as those derived by Coffman, Kundu, 
and Wootters [23], hold only for qubit systems [24]. Also, not all measures of entanglement satisfy this relation. It turns out that there exists other versions of monogamy inequalities based on squashed entanglement [25], distillable entanglement [26], etc. Since most measures of entanglement are difficult to calculate, analytical bounds are quite desirable.

Recently, we found an analytical lower bound of concurrence [27], which satisfies a monogamy inequality. We also found the monogamy of negativity [28]. Interestingly, for Gaussian states, the monogamy of negativity also holds [29] and there exists an even stronger bound [30]. There was an attempt to use convex-roof extended negativity [31] to define a monogamy entanglement for three-qubit states, but it is unclear whether such a property is true for pure tripartite states with arbitrary dimensions. As for the monogamy of negativity itself [28], it is not clear either.

In this paper, we will establish a set of much more general constraints on entanglementsharing in multipartite states, which are computable. These complement the constraints given in Ref. [28]. Specifically, Section 2 introduces the basic concepts of monogamy of concurrence, negativity, and realignment. Section 3 proves the more general monogamy of negativity and realignment, and computable inequalities for any three-qubit states. Section 4 provides applications, methods for testing these inequalities through measurements on sets of qubits, and Section 5 concludes.

\section{Monogamy of entanglement}

For a mixed state $\rho_{A B}$, one well-known measure of entanglement is the EOF [8] defined by $E\left(\rho_{A B}\right)=\min _{\left\{p_{i},\left|\psi_{i}\right\rangle\right\}} \sum_{i} p_{i} E\left(\left|\psi_{i}\right\rangle\right)$ for all possible ensemble realizations $\rho_{A B}=\sum_{i} p_{i}\left|\psi_{i}\right\rangle\left\langle\psi_{i}\right|$. Here $p_{i} \geq 0, \sum_{i} p_{i}=1$, and $E\left(\left|\psi_{A B}\right\rangle\right)=-\operatorname{Tr} \rho_{A} \log _{2} \rho_{A}$ with $\rho_{A}=\operatorname{Tr}_{B}\left|\psi_{A B}\right\rangle\left\langle\psi_{A B}\right|$. For twoqubit states, the EOF can be expressed as a function of concurrence defined by [8]

$$
\mathcal{C}(\rho) \equiv \max \left\{0, \sqrt{\lambda_{1}}-\sqrt{\lambda_{2}}-\sqrt{\lambda_{3}}-\sqrt{\lambda_{4}}\right\},
$$

where $\lambda_{1}, \ldots, \lambda_{4}$ are the singular values of the matrix $\rho\left(\sigma_{y} \otimes \sigma_{y}\right) \rho^{*}\left(\sigma_{y} \otimes \sigma_{y}\right)$ in nonincreasing order, the notation of $*$ stands for complex conjugation in a particular basis, and $\sigma_{y}$ is the standard Pauli spin matrix. For a pure three-qubit state $\rho_{A B C}$, there exists an inequality in terms of concurrence which provides a quantification of three-qubit entanglement [23]

$$
\mathcal{C}_{A B}^{2}+\mathcal{C}_{A C}^{2} \leq \mathcal{C}_{A: B C}^{2}
$$

where $\mathcal{C}_{A B}$ and $\mathcal{C}_{A C}$ are the concurrences of the mixed states $\rho_{A B}=\operatorname{Tr}_{\mathrm{C}}\left(|\phi\rangle_{\mathrm{ABC}}\langle\phi|\right)$ and $\rho_{A C}=\operatorname{Tr}_{\mathrm{B}}\left(|\phi\rangle_{\mathrm{ABC}}\langle\phi|\right)$, respectively, and $\mathcal{C}_{A: B C}=2 \sqrt{\operatorname{det} \rho_{A}}$ with $\rho_{A}=\operatorname{Tr}_{\mathrm{BC}}\left(|\phi\rangle_{\mathrm{ABC}}\langle\phi|\right)$. We refer to this as the CKW inequality [23]. Based on this inequality in Eq. (2) the three-tangle is defined as

$$
\tau_{A B C}=\mathcal{C}_{A: B C}^{2}-\mathcal{C}_{A B}^{2}-\mathcal{C}_{A C}^{2},
$$

which characterizes three-way entanglement of the state and is an entanglement monotone [32]. For example, quantified by the three-tangle, the state $|G H Z\rangle=\frac{1}{\sqrt{2}}(|000\rangle+|111\rangle)$ has only three-way entanglement, while the state $|W\rangle=\frac{1}{\sqrt{3}}(|100\rangle+|010\rangle+|001\rangle)$ has only twoway entanglement. For general three-qubit mixed states $\rho_{A B C}$, the three-tangle is defined as

$$
\tau_{A B C}=\min \left[\mathcal{C}_{A: B C}^{2}\right]-\mathcal{C}_{A B}^{2}-\mathcal{C}_{A C}^{2},
$$


where

$$
\min \left[\mathcal{C}_{A: B C}^{2}\right]=\min _{\left\{p_{i},\left|\psi_{i}\right\rangle\right\}}\left[\sum_{i} p_{i} \mathcal{C}_{A: B C}^{2}\left(\left|\psi_{i}\right\rangle\right)\right]
$$

denotes the minimum over all pure-state decompositions such that $\rho_{A: B C}=\sum p_{i}\left|\psi_{i}\right\rangle\left\langle\psi_{i}\right|$.

Two other important measures of entanglement are the negativity and the realignment. The negativity is based on trace norm of the partial transpose $\rho^{T_{A}}$ of a bipartite state $\rho_{A B}$, and in fact it is a quantitative version of the positive partial transposition (PPT) criterion for separability $[6,7]$. For a bipartite state $\rho$ the partial transpose with respect to subsystem $A$ is

$$
\left(\rho^{T_{A}}\right)_{i j, k l}=\rho_{k j, i l},
$$

and the negativity is defined by

$$
\mathcal{N}=\frac{\left\|\rho^{T_{A}}\right\|-1}{2}
$$

where the trace norm $\|R\|$ is given by $\|R\|=\operatorname{Tr} \sqrt{R^{\dagger} R}$. The negativity can also be seen to be equal to the sum of the negative eigenvalues of $\rho^{T_{A}}$. The realignment, or cross-norm $[9,10]$ can detect entanglement for which the negativity fails. Similar to the negativity, it is defined in terms of a relabeling

$$
\mathcal{R}(\rho)_{i j, k l}=\rho_{i k, j l},
$$

which results in the following measure

$$
\mathcal{R}=\max \left\{\frac{\|\mathcal{R}(\rho)\|-1}{2}, 0\right\} .
$$

For simplicity, we refer to the quantity $\mathcal{R}$ as the realignment. We take the maximum in the above equation since the first term in the bracket can be negative for some states. Note that $\mathcal{N}=0$ is necessary for separability of any bipartite states and is also sufficient for $2 \otimes 2$ and $2 \otimes 3$ states. But the realignment $\mathcal{R}=0$ is a necessary condition of separability for all bipartite states.

By using the monogamy of concurrence (2), the monogamy of negativity and realignment has been proven $[28,33]$. Thus, for any pure three-qubit states $\rho_{A B C}$, we have

$$
\mathcal{N}_{A B}^{2}+\mathcal{N}_{A C}^{2} \leq \mathcal{N}_{A: B C}^{2},
$$

and

$$
\mathcal{R}_{A B}^{2}+\mathcal{R}_{A C}^{2} \leq \mathcal{R}_{A: B C}^{2} .
$$

The objective of this paper is to provide a set of general and computable constraints on entanglement sharing for multipartite quantum states. To do this, we first note that the general monogamy inequalities for pure multi-qubit states $\rho_{A_{1} A_{2} \ldots A_{n}}$ in terms of concurrence, negativity, and realignment. The concurrence satisfies

$$
\mathcal{C}_{A_{1} A_{2}}^{2}+\mathcal{C}_{A_{1} A_{3}}^{2}+\cdots+\mathcal{C}_{A_{1} A_{n}}^{2} \leq \mathcal{C}_{A_{1}: A_{2} A_{3} \ldots A_{n}}^{2},
$$

the negativity satisfies

$$
\mathcal{N}_{A_{1} A_{2}}^{2}+\mathcal{N}_{A_{1} A_{3}}^{2}+\cdots+\mathcal{N}_{A_{1} A_{n}}^{2} \leq \mathcal{N}_{A_{1}: A_{2} A_{3} \ldots A_{n}}^{2},
$$


and the realignment also satisfies

$$
\mathcal{R}_{A_{1} A_{2}}^{2}+\mathcal{R}_{A_{1} A_{3}}^{2}+\cdots+\mathcal{R}_{A_{1} A_{n}}^{2} \leq \mathcal{R}_{A_{1}: A_{2} A_{3} \ldots A_{n}}^{2},
$$

where $\mathcal{C}_{A_{1} A_{i}}, \mathcal{N}_{A_{1} A_{i}}$, and $\mathcal{R}_{A_{1} A_{i}}$ denote two-qubit quantities and $\mathcal{C}_{A_{1}: A_{2} A_{3} \ldots A_{n}}^{2}, \mathcal{N}_{A_{1}: A_{2} A_{3} \ldots A_{n}}^{2}$, and $\mathcal{R}_{A_{1}: A_{2} A_{3} \ldots A_{n}}^{2}$ are the bipartite entanglement measured by the respective quantities across the (bi)partition $A_{1}: A_{2} A_{3} \ldots A_{n}$. The detailed proof of the inequalities (12) and (13) can be found in $[23,34,28]$. The verification of the inequality (14) follows immediately from the work of $[28,33]$, so is omitted here.

\section{Computable monogamy inequality in terms of negativity and realignment}

It should be emphasized that the inequalities (12-14) are limited to bipartite subsystems, i.e., $A_{i}$ is a single qubit, and do not provide the extent to which the general monogamy inequality applies. This is important because it has been shown that the monogamy of concurrence does not generally hold for higher-dimensional quantum states [24]. In view of the difficulty of analytically calculating entanglement of bipartite states with dimension greater than 2 , for pure multi-qubit states, we propose a more general monogamy constraint on the corresponding distribution of entanglement. The most important thing is that it is computable, as shown in the following theorem, one of two main results of this paper.

Theorem 1: For pure multi-qubit states $\rho_{A B C \ldots D E}$ where $A, B, C, \ldots, D$ stand for single qubits, respectively, while $E$ a collection of any number of qubits, i.e., $E=\left(E_{1} E_{2} \ldots E_{l}\right)$ where $E_{1}, E_{2}, \ldots, E_{l}$ are qubits, the negativity and realignment satisfy the following inequalities

$$
\mathcal{N}_{A B}^{2}+\mathcal{N}_{A C}^{2}+\cdots+\mathcal{N}_{A D}^{2}+\mathcal{N}_{A E}^{2} \leq \mathcal{N}_{A: B C \ldots D E}^{2}
$$

and

$$
\mathcal{R}_{A B}^{2}+\mathcal{R}_{A C}^{2}+\cdots+\mathcal{R}_{A D}^{2}+\mathcal{R}_{A E}^{2} \leq \mathcal{R}_{A: B C \ldots D E}^{2} .
$$

Proof: The proof is straightforward and stems from the fact that both the negativity and the realignment are lower bounds of the concurrence. For a pure bipartite state $\rho_{F_{1} F_{2}}=$ $\left|\Psi_{F_{1} F_{2}}\right\rangle\left\langle\Psi_{F_{1} F_{2}}\right|$ in $2 \otimes d$ dimensions, the system has the Schmidt form

$$
\left|\Psi_{F_{1} F_{2}}\right\rangle=\sqrt{\lambda_{1}}\left|e_{1} f_{1}\right\rangle+\sqrt{\lambda_{2}}\left|e_{2} f_{2}\right\rangle,
$$

where $\sqrt{\lambda_{i}}$ are Schmidt coefficients and $\left|e_{i}\right\rangle$ and $\left|f_{i}\right\rangle$ are orthonormal bases for $\mathcal{H}_{F_{1}}$ and $\mathcal{H}_{F_{2}}$, respectively. It is shown that $[16,28]$

$$
\mathcal{C}_{F_{1} F_{2}}=2 \mathcal{N}_{F_{1} F_{2}}=2 \mathcal{R}_{F_{1} F_{2}}=2 \sqrt{\lambda_{1} \lambda_{2}} .
$$

In a similar way, we have

$$
\mathcal{C}_{A: B C \ldots D E}=2 \mathcal{N}_{A: B C \ldots D E}=2 \mathcal{R}_{A: B C \ldots D E} .
$$

Now if $\rho_{F_{1} F_{2}}$ is a mixed state, then [16]

$$
2 \mathcal{N}_{F_{1} F_{2}} \leq \mathcal{C}_{F_{1} F_{2}},
$$

and

$$
2 \mathcal{R}_{F_{1} F_{2}} \leq \mathcal{C}_{F_{1} F_{2}}
$$


Y.-C. Ou and M.S. Byrd 5

Table 1. Values of the residual entanglement defined by the $\tau_{A B C}$ and $\pi_{A B C}$ different classes.

\begin{tabular}{ccc}
\hline \hline Class & $\tau_{A B C}$ & $\pi_{A B C}$ \\
\hline A-B-C & 0 & 0 \\
\hline A-BC & 0 & 0 \\
\hline B-AC & 0 & 0 \\
\hline C-AB & 0 & 0 \\
\hline W & 0 & $>0$ \\
\hline GHZ & $>0$ & $>0$ \\
\hline \hline
\end{tabular}

Note that if $F_{1}$ is not two-dimensional, some extra constant factors will appear in the expressions on the left-hand sides of Eqs. (20) and (21).

For either pure or mixed states $\rho_{G H K}$ in $2 \otimes 2 \otimes 2^{n-2}$ dimensions we have [34]

$$
\mathcal{C}_{G H}^{2}+\mathcal{C}_{G K}^{2} \leq \mathcal{C}_{G: H K}^{2}
$$

In order to obtain our desired result, we partition $K$ into two parts, i.e., $K_{1}$ and $K_{2} . K_{1}$ is a single qubit while $K_{2}$ is a collection of $n-3$ qubits. Therefore, according to Eq. (22), the following inequality holds

$$
\mathcal{C}_{G H}^{2}+\mathcal{C}_{G K_{1}}^{2}+\mathcal{C}_{G K_{2}}^{2} \leq \mathcal{C}_{G: H K}^{2}
$$

Analogously, successively applying Eqs. (22) and (23) to the pure state $\rho_{A B C \ldots D E}$ gives

$$
\mathcal{C}_{A B}^{2}+\mathcal{C}_{A C}^{2}+\cdots+\mathcal{C}_{A D}^{2}+\mathcal{C}_{A E}^{2} \leq \mathcal{C}_{A: B C \ldots D E}^{2},
$$

such that the proofs of (15) and (16) can be finished by considering (19-21) and (24).

We remark that Theorem 1 would be much more general if $A, B, C, \ldots D$ contained an arbitrary number of qubits, respectively. However, since it is much easier to calculate $\mathcal{N}_{A E}$ and $\mathcal{R}_{A E}$ than $\mathcal{C}_{A E}$, we believe these may provide a certain quantification of the correlations, which could be valuable in quantum cryptographic protocols and among other uses.

Note that our result is not limited to pure multi-qubit states. For mixed states these monogamy inequalities also hold when the right-hand side is minimized over all pure-state decompositions as in (5). This can be done using the convexity of negativity and realignment. However, the quantitative evaluation then becomes intractable for large systems. By decomposing a higher-dimensional Hilbert space into lower-dimensional spaces, useful bounds on entanglement between different parties can be obtained.

For a tripartite state, the smallest Hilbert space is $2 \otimes 2 \otimes 2$ dimensional. The bound for the entanglement gives some indications of the distribution of entanglement of the whole state. For example, for either pure or mixed three-qubit states $\rho_{A B C}$, the concurrence between $A$ and $B$ is limited by the one between $C$ and $A B$ [35], i.e.,

$$
\mathcal{C}_{A B} \leq \frac{1}{2}\left(1+\sqrt{1-\mathcal{C}_{C: A B}^{2}}\right)
$$

Given Eqs. (20), (21), and (25) we can also state the following theorem concerning the monogamy of entanglement as measured by the negativity and realignment.

Theorem 2: For any three-qubit state $\rho_{A B C}$, the entanglement between $A$ and $B$, and the one between $C$ and $A B$ satisfy

$$
\mathcal{N}_{A B} \leq \frac{1}{4}\left(1+\sqrt{1-4 \mathcal{N}_{C: A B}^{2}}\right)
$$




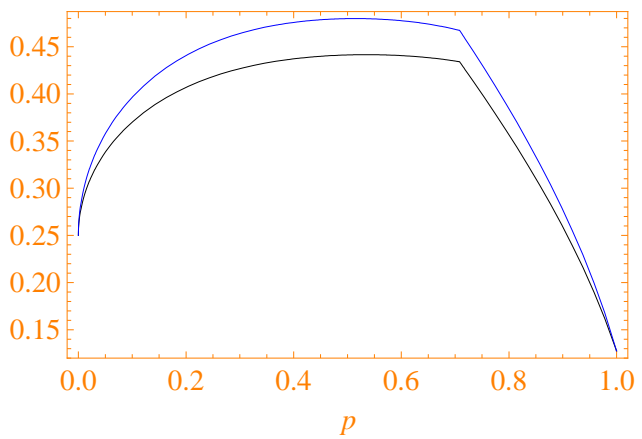

Fig. 1. Plot of $\tau_{\mathcal{N}}$ (lower curve) and $\tau_{\mathcal{R}}$ (upper curve) for the states (30) as a function of $p$.

and

$$
\mathcal{R}_{A B} \leq \frac{1}{4}\left(1+\sqrt{1-4 \mathcal{R}_{C: A B}^{2}}\right) .
$$

Again, we emphasize the computability of these results.

\section{Application and discussion}

In this section, several examples are discussed which show the utility of the monogamy inequalities established above.

\subsection{Examples}

To compare various classes of entangled states, we recall the definition of the residual entanglement

$$
\pi_{A B C}=\mathcal{N}_{A: B C}^{2}-\mathcal{N}_{A B}^{2}-\mathcal{N}_{A C}^{2} .
$$

This quantity is greater than zero for pure three-qubit states $\rho_{A B C}$, for the $W$-class and $G H Z$-class [28], while the quantification of entanglement given by the three-tangle in Eq. (3) is greater than zero only for the $G H Z$-class [32]. Although it has been demonstrated in Ref. [28], we provide a comparison of the inequalities in Table 1 . Note that all pure three-qubit states can be sorted into six classes through stochastic local operation and classical communication (SLOCC) [32]. (1) $A-B-C$ class including product states; (2) $A-B C$, (3) $B-A C$, and (4) $C-A B$ classes including only bipartite entangled states; (5) $W$ and (6) GHZ classes including so-called genuine tripartite entangled states. It is evident that one can distinguish the two classes of fully entangled states with the help of $\tau_{A B C}$ and $\pi_{A B C}$. Furthermore, we have numerically verified that the monogamy inequality of realignment, Eq. (11), is also strict for pure three-qubit states. Given this, we have the following conjecture.

\subsection{A Conjecture}

Conjecture: For a pure multipartite state $\rho_{A B C D \ldots}$, if the particle $A$ is entangled with at least two other particles, the monogamy inequalities (15-16) are strict.

On the other hand, the inequalities Eq. (26) and Eq. (27) have similar properties for pure states. For a pure state $\rho_{A B C}$ in the $G H Z$-class, it is obvious that the two inequalities are strict since $\mathcal{N}_{A B}=\mathcal{R}_{A B}=0$ for this class while the right-hand sides of Eq. (26) and Eq. (27) 
are not less than 0.25. Some $W$-class states saturate the inequality Eq. (25), and since it has been shown that $2 \mathcal{N}_{A B}<\mathcal{C}_{A B}$ [28], the inequality Eq. (26) is also strict for this class. Likewise, the inequality Eq. (27) is strict for the $W$-class. As for the other four classes, only for the following entangled states

$$
|\phi\rangle_{A B} \otimes|\phi\rangle_{C}=U_{A} \otimes U_{B} \otimes I \frac{1}{\sqrt{2}}(|00\rangle+|11\rangle)_{A B} \otimes|\phi\rangle_{C},
$$

where $U_{A}, U_{B} \in U(2)$, do the inequalities Eq. (26) and Eq. (27) become equalities.

Now let us investigate the rank-2 mixed three-qubit states

$$
\rho(p)=p|W\rangle\langle W|+(1-p)| G H Z\rangle\langle G H Z|,
$$

with $p \in[0,1]$. We define $\tau_{\mathcal{N}}$ and $\tau_{\mathcal{R}}$ to be the difference between the left-hand side and right-hand side of Eq. (26) and Eq. (27) respectively, i.e.,

$$
\tau_{\mathcal{N}}=\frac{1}{4}\left(1+\sqrt{1-4 \mathcal{N}_{C: A B}^{2}}\right)-\mathcal{N}_{A B}
$$

and

$$
\tau_{\mathcal{R}}=\frac{1}{4}\left(1+\sqrt{1-4 \mathcal{R}_{C: A B}^{2}}\right)-\mathcal{R}_{A B}
$$

We plot these in Fig. 1 for the states given in Eq. (30). From Fig. 1, we can see that the monogamy of negativity Eq. (31) and realignment Eq. (32) provide different constraints for these states, in this sense, generally they are inequivalent. For some states however, they are complementary. In contrast to Eq. (31) and Eq. (32), for mixed states the monogamy constraints for distributed entanglement Eq. (4) and Eq. (25) are very difficult to calculate.

\subsection{Experimental Determination}

For applications of this work, we provide a direct route to experimental confirmation of these inequalities for sets of qubits. We do this by noting that the components of the polarization vector (a.k.a the generalized coherence vector, or Bloch vector representation [36, 37, 38, 39]) are experimentally measurable quantities. We note that several important examples of the concurrence [38], the realignment [9], and the negativity [40], have already been expressed in terms of the polarization vector.

To be specific, the square of the concurrence for any two two-state subsystems, $A, B$ can be expressed in terms of the square roots of the eigenvalues of the matrix

$$
M=\rho_{A B} \tilde{\rho}_{A B},
$$

which we will let be $\lambda_{1}, \lambda_{2}, \lambda_{3}, \lambda_{4}$ with $\lambda_{i} \geq \lambda_{i+1}$. The square of the concurrence for two subsystems $A$ and $B$ is

$$
\begin{aligned}
\mathcal{C}_{A B}^{2}= & \left(\lambda_{1}-\lambda_{2}-\lambda_{3}-\lambda_{4}\right)^{2} \\
= & \lambda_{1}^{2}+\lambda_{2}^{2}+\lambda_{3}^{2}+\lambda_{4}^{2} \\
& -2 \lambda_{1} \lambda_{2}-2 \lambda_{1} \lambda_{3}-2 \lambda_{1} \lambda_{4} \\
& +2 \lambda_{2} \lambda_{3}+2 \lambda_{2} \lambda_{4}+2 \lambda_{3} \lambda_{4} .
\end{aligned}
$$


Note that the following inequality holds which is useful for the inequalities provided later:

$$
\lambda_{1} \lambda_{2}+\lambda_{1} \lambda_{3}+\lambda_{1} \lambda_{4} \geq \lambda_{2} \lambda_{3}+\lambda_{2} \lambda_{4}+\lambda_{3} \lambda_{4}
$$

In fact each term on the left is, taking the terms in order, greater than those on the right.

Now, dropping the negative terms, and adding a few

$$
C_{A B} \leq \operatorname{Tr}(M)+2\left[S_{2}(M)\right]^{1 / 2}
$$

where $S_{2}(M)=(1 / 2)\left[(\operatorname{Tr}(M))^{2}-\operatorname{Tr}\left(M^{2}\right)\right]$ [38]. Dropping the positive terms, and subtracting a few, we obtain

$$
C_{A B} \geq \operatorname{Tr}(M)-2\left[S_{2}(M)\right]^{1 / 2}
$$

Clearly this latter inequality is only meaningful when both the concurrence and the right-hand side are both greater than zero.

Now $\rho_{A B}$ and $\tilde{\rho}_{A B}$, for qubits, can be expressed in terms of the polarization vector as

$$
\rho_{A B}=\frac{1}{4}\left(I+\vec{n}_{A} \cdot \vec{\sigma} \otimes I+I \otimes \vec{n}_{B} \cdot \vec{\sigma}+\sum_{\alpha, \beta} c_{\alpha \beta} \sigma_{\alpha} \otimes \sigma_{\beta}\right),
$$

and

$$
\tilde{\rho}_{A B}=\frac{1}{4}\left(I-\vec{n}_{A} \cdot \vec{\sigma} \otimes I-I \otimes \vec{n}_{B} \cdot \vec{\sigma}+\sum_{\alpha, \beta} c_{\alpha \beta} \sigma_{\alpha} \otimes \sigma_{\beta}\right) .
$$

One can multiply these two and to obtain $M$ and thus all bounds on the concurrence given in this article in terms of experimentally available quantities. The required measurements only require, at most, sets of two-body interactions.

In the case that the density operator under consideration has single-particle density operators which are completely mixed, the bounds simplify dramatically. The matrix $\tilde{\rho}$ becomes equal to $\rho$,

$$
\tilde{\rho}_{A B}=\rho_{A B}=\frac{1}{4}\left(I+\sum_{\alpha, \beta} c_{\alpha \beta} \sigma_{\alpha} \otimes \sigma_{\beta}\right) .
$$

The bounds above can be computed and measured by well-known methods [41].

Furthermore, for the two-qubit case that the local density operators are completely mixed, the realignment, or cross-norm, can also be readily computed in terms of measurable quantities without relying on bounds. Since the entanglement measures are invariant under local unitary transformations, local unitary transformations can be used to diagonalize the correlation matrix $c_{\alpha \beta}$, to get $c_{\alpha \alpha}$ and the realignment gives

$$
\mathcal{R}(\rho)=\frac{1}{2}\left(1+\sum_{\alpha}\left|c_{\alpha \alpha}\right|\right) .
$$

These are themselves measurable quantities, as are the $c_{\alpha \beta}$ before diagonalization. Similarly the negativity for the two-qubit state can be found in terms of the correlation matrix 
components by direct computation,

$$
\begin{aligned}
\mathcal{N}\left(\rho_{A B}\right)= & \frac{1}{2}\left(\left|\frac{1}{4}+c_{33}+c_{11}-c_{22}\right|\right. \\
& +\left|\frac{1}{4}+c_{33}-c_{11}+c_{22}\right|+\left|\frac{1}{4}-c_{33}+c_{11}+c_{22}\right| \\
& \left.+\left|\frac{1}{4}-c_{33}-c_{11}-c_{22}\right|\right) .
\end{aligned}
$$

These expressions provide a direct route to experimental determination of the bounds provided in this article using the two-particle correlations.

\section{Conclusions}

Here we have derived monogamy inequalities in terms of negativity and realignment. Since these two measures of entanglement are computable, these inequalities provide a quantitative evaluation of constraints on entanglement-sharing for multipartite states. Most importantly, Eq.(15) and Eq.(16) provide inequalities which are not necessarily limited to bipartite systems and are able to give more information about the possibilities for distributing entanglement. The importance of these also lie in the fact they allow us to investigate the distribution of entanglement based on different partitions of many-qubit systems in condensed matter physics. We also derive computable monogamy inequalities for any three-qubit states. Furthermore, experimental determination of these bounds may be accomplished given the expressions here. In the near future, studying the entanglement in three-qubit states as done in [42] through the results in this paper is worthwhile, since different monogamy inequalities can lead to different residual entanglement. We believe our work will greatly aid in the understanding of the physical implications of these inequalities.

The most appealing feature of Eq. (26) and Eq. (27) is that these quantities are computable entanglement-sharing constraints for mixed three-qubit states.

\section{Acknowledgements}

This material is based upon work supported by the National Science Foundation under Grant No. 0545798. We thank Shao-Ming Fei and Heng Fan for valuable discussions.

\section{References}

1. A. K. Ekert (1991), Quantum cryptography based on Bells theorem, Phys. Rev. Lett. 67, pp. 661-663.

2. C. H. Bennett, G. Brassard, C. Crepeau, R. Jozsa, A. Peres and W. K. Wootters (1993), Teleporting an unknown quantum state via dual classical and Einstein-Podolsky-Rosen channels, Phys. Rev. Lett. 70, pp. $1895-1899$.

3. R. Horodecki, P. Horodecki, M. Horodecki, and K. Horodecki (2009), Quantum entanglement, Rev. Mod. Phys. 81, pp. 865-942.

4. O. Guhne and G. Toth (2009), Entanglement detection, Physics Reports, 474, pp. 1-75.

5. R. F. Werner (1989), Quantum states with Einstein-Podolsky-Rosen correlations admitting a hidden-variable model, Phys. Rev. A 40, pp. 4277-4281.

6. A. Peres (1996), Separability criterion for density matrices, Phys. Rev. Lett. 77, pp. 1413-1415. 
7. M. Horodecki, P. Horodecki and R. Horodecki (1996), Separability of mixed states: necessary and sufficient conditions, Phys. Lett. A 223, pp. 1-8.

8. W. K. Wootters (1998), Entanglement of formation of an arbitrary state of two qubits, Phys. Rev. Lett. 80, pp. 2245-2248.

9. O. Rudolph (2002), Further results on the cross norm criterion for separability , arXiv:quant$\mathrm{ph} / 0202121$.

10. K. Chen and L. A. Wu (2003), A matrix realignment method for recognizing entanglement, Qu. Inf. \& Comp. 3, pp. 193-202.

11. G. Toth and O. Guhne (2005), Detecting genuine multipartite entanglement with two local measurements, Phys. Rev. Lett. 94, pp. 060501.

12. O. Guhne, P. Hyllus, O. Gittsovich, and J. Eisert (2007), Covariance matrices and the separability problem, Phys. Rev. Lett. 99, pp. 130504.

13. C. J. Zhang, Y. S. Zhang, S. Zhang, and G. C. Guo (2008), Entanglement detection beyond the computable cross-norm or realignment criterion, Phys. Rev. A 77, pp. 060301.

14. J. Zhang, C. W. Li, J. W. Wu, R. B. Wu, and T. J. Tarn (2006), Quasimultipartite entanglement measure based on quadratic functions, Phys. Rev. A 73, pp. 022319.

15. F. Mintert, M. Kus, and A. Buchleitner (2004), Concurrence of mixed bipartite quantum states in arbitrary dimensions, Phys. Rev. Lett. 92, pp. 167902.

16. K. Chen, S. Albeverio, S. M. Fei (2005), Concurrence of arbitrary dimensional bipartite quantum states, Phys. Rev. Lett. 95, pp. 040504.

17. H. Breuer (2006), Separability criteria and bounds for entanglement measures, J. Phys. A 39, pp. 11847-11860.

18. J. I. de Vicente (2007), Lower bounds on concurrence and separability conditions, Phys. Rev. A 75 , pp. 052320.

19. D. Li, X. Li, H. Huang, and X. Li (2007), Stochastic local operations and classical communication invariant and the residual entanglement for $n$ qubits, Phys. Rev. A 76, pp. 032304.

20. X. H. Gao, S. M. Fei, and K. Wu (2006), Lower bounds of concurrence for tripartite quantum systems, Phy. Rev. A 74, pp. 050303.

21. S. M. Fei and X. Li-Jost (2006), R function related to entanglement of formation, Phys. Rev. A 73, pp. 024302.

22. W. Song, N. L. Liu, and Z. B. Chen (2007), Bounds on the multipartite entanglement of superpositions, Phys. Rev. A 76, pp. 054303.

23. V. Coffman, J. Kundu, and W. K. Wootters (2000), Distributed entanglement, Phys. Rev. A 61, pp. 052306.

24. Y. C. Ou (2007), Violation of monogamy inequality for higher-dimensional objects, Phys. Rev. A 75, pp. 034305.

25. M. Christandl and A. Winter (2004), "Squashed entanglement": An additive entanglement measure, J. Math. Phys. 45, pp. 829-840.

26. M. Koashi and A. Winter (2004), Monogamy of quantum entanglement and other correlations, Phys. Rev. A 69, pp. 022309.

27. Y. C. Ou, H. Fan, and S. M. Fei (2008), Proper monogamy inequality for arbitrary pure quantum states, Phys. Rev. A 78, pp. 012311.

28. Y. C. Ou and H. Fan (2007), Monogamy inequality in terms of negativity for three-qubit states, Phys. Rev. A 75, pp. 062308.

29. T. Hiroshima, G. Adesso, and F. Illuminati (2007), Monogamy inequality for distributed gaussian entanglement, Phys. Rev. Lett. 98, pp. 050503.

30. G. Adesso and F. Illuminati (2008), Genuine multipartite entanglement of symmetric Gaussian states: Strong monogamy, unitary localization, scaling behavior, and molecular sharing structure, Phys. Rev. A 78, pp. 042310.

31. J. S. Kim, A. Das, and B. C. Sanders (2009), Entanglement monogamy of multipartite higherdimensional quantum systems using convex-roof extended negativity, Phys. Rev. A 79, pp. 012329 . 
32. W. Dur, G. Vidal, and J. I. Cirac (2000), Three qubits can be entangled in two inequivalent ways, Phys. Rev. A 62, pp. 062314.

33. H. Fan, Y. C. Ou, and V. Roychowdhury (2007), Entangled multi-qubit states without highertangle, arXiv:quant-ph/0707.1578.

34. T. J. Osborne and F. Verstraete (2006), General monogamy inequality for bipartite qubit entanglement, Phys. Rev. Lett. 96, pp. 220503.

35. J. H. Huang and S. Y. Zhu (2008), Entanglement monogamy in a three-qubit state, Phys. Rev. A 78, pp. 012325.

36. G. Mahler and V. A. Weberruss, Quantum Networks: Dynamics of Open Nanostructures (Springer Verlag, Berlin, 1998), 2nd ed.

37. L. Jakobczyk and M. Siennicki (2001), Geometry of Bloch vectors in two-qubit system, Phys. Lett. A 286, pp. 383-390.

38. M. S. Byrd and N. Khaneja (2003), Characterization of the positivity of the density matrix in terms of the coherence vector representation, Phys. Rev. A 68, pp. 062322.

39. G. Kimura (2003), The Bloch vector for N-level systems, Phys. Lett. A 314, pp. 339-349.

40. M.S. Byrd and G.K. Brennen (2008), General depolarized pure states: Identification and properties, Phys. Lett. A 372, pp. 1770-1782.

41. T. A. Brun (2004), Measuring polynomial functions of states, Qu. Info. \& Comp. 4, pp. 401-408.

42. R. Lohmayer, A. Osterloh, J. Siewert, and A. Uhlmann (2006), entangled three-qubit states without concurrence and three-tangle, Phys. Rev. Lett. 97, pp. 260502. 